\title{
Frequency stabilization and actuator characterization of an ytterbium-doped distributed-feedback fiber laser for LISA
}

\author{
Michael Tröbs, ${ }^{1, *}$ Luigi d'Arcio, ${ }^{2}$ Gerhard Heinzel, ${ }^{1}$ and Karsten Danzmann ${ }^{1}$ \\ ${ }^{1}$ AEI Hannover, (MPI für Gravitationsphysik und Leibniz Universität Hannover), Callinstr. 38, \\ 30167 Hannover, Germany \\ ${ }^{2}$ European Space Agency, postbus 299, 2200 AG Noordwijk, The Netherlands \\ *Corresponding author: michael.troebs@aei.mpg.de
}

Received January 30, 2009; revised March 20, 2009; accepted March 28, 2009; posted April 1, 2009 (Doc. ID 106737); published April 30, 2009

\begin{abstract}
We have investigated an ytterbium-doped distributed-feedback fiber master oscillator power amplifier system emitting $1 \mathrm{~W}$ and its suitability for the space-borne interferometric gravitational-wave detector Laser Interferometer Space Antenna (LISA). For this purpose we measured the laser system's free-running frequency noise, characterized its frequency actuator, and implemented a robust frequency stabilization. Up to $100 \mathrm{~Hz}$ Fourier frequency the free-running frequency, noise was comparable to that of a nonplanar ring oscillator. The first resonance of the actuator was at $32 \mathrm{kHz}$ with a quality factor of 26 and a delay of $20 \mu \mathrm{s}$. The frequency lock to a thermally shielded Fabry-Perot cavity was stable over many hours and fulfilled the LISA requirements. (C) 2009 Optical Society of America
\end{abstract}

OCIS codes: $140.3425,140.3510,140.3570$.

\section{INTRODUCTION}

The space-based gravitational-wave detector Laser Interferometer Space Antenna (LISA) [1] requires laser sources with extreme frequency stability. The most promising laser system candidates are a stand-alone highpower nonplanar ring oscillator (NPRO) operating at $1064 \mathrm{~nm}[2,3]$ or a fiber amplifier seeded by a low-power NPRO [4]. Advantages of the latter system are the high efficiency of the fiber amplifier and the power scalability of the overall laser system. In a master-oscillator poweramplifier concept, the use of a fiber oscillator is obviously attractive in terms of a compact and rugged all-fiber system, provided the fiber oscillator shows similar or lower free-running and stabilized frequency noise as an NPRO.

Since LISA requires active frequency stabilization and an offset-phase lock of its lasers, a frequency actuator is required. The lasers on board the LISA satellites will be frequency locked to an external reference cavity or to the mean armlength [5], or, to a remote laser in a transponder scheme [1]. For the design of the frequency-stabilization control loop, knowledge of the frequency actuator transfer function is required.

To date, frequency stabilization results of erbium-doped fiber lasers at $1.5 \mu \mathrm{m}$ wavelength have been reported. A standing-wave laser with grating feedback and coupled cavities has been frequency locked to the side of acetylene absorption lines [6], a ring laser has been frequency locked to an external fiber Fabry-Perot resonator [7] using the Pound-Drever-Hall technique [8], a distributedfeedback (DFB) laser has been frequency locked to a Mach-Zehnder interferometer [9], and a distributed Bragg reflector (DBR) laser has been locked to an unbalanced-arm scanning Michelson interferometer [10].
A fiber ring laser has been frequency stabilized by injection locking [11]. The free-running frequency noise of two identical DBR lasers has been measured for Fourier frequencies up to $100 \mathrm{kHz}$ [12]. Frequency noise above $1 \mathrm{~Hz}$ and the frequency actuator transfer function of a DBR fiber laser have been reported [13]. Frequency noise from a few $\mathrm{Hz}$ to approximately $60 \mathrm{kHz}$ for fiber lasers emitting at $1064 \mathrm{~nm}, 1550 \mathrm{~nm}$, and $1893 \mathrm{~nm}$ have been reported [14]. Recently, frequency-stabilized fiber lasers have been used in precision metrology [15] and for optical clock transfer [16].

In contrast to the lasers discussed above, LISA requires a laser emitting at least $1 \mathrm{~W}$ at $1064 \mathrm{~nm}$ wavelength. We have investigated an ytterbium-doped distributedfeedback fiber master oscillator power amplifier system emitting $1 \mathrm{~W}$ and its suitability for LISA. In Section 2 we discuss the free-running frequency noise of the laser system in comparison to that of a NPRO for Fourier frequencies from below $0.1 \mathrm{mHz}$ up to $1 \mathrm{MHz}$. Section 3 reports on the efficiency and transfer function of the frequency actuator.

\section{FREQUENCY NOISE}

An ytterbium-doped DFB fiber laser [Boostik Y10 by Koheras A/S (Denmark)] was used. It consisted of a master oscillator that was amplified by two double-clad fiber amplifiers. The oscillator and the amplifiers were pumped at $980 \mathrm{~nm}$ by laser diodes and emitted at $1064 \mathrm{~nm}$. Singlefrequency operation and frequency tuning were achieved by a tunable grating written into the active fiber. The laser emitted up to $1 \mathrm{~W}$ from a polarization-maintaining fiber and was equipped with a piezo-electric element (PZT), 
which strained the fiber grating and tuned the laser frequency when a voltage was applied. This analog modulation input was used for frequency stabilization as described below.

Figure 1 shows the setup that was used for measuring the free-running frequency noise, frequency stabilization, and actuator characterization of the fiber laser. The light emitted from the fiber laser was collimated by a lens and reflected from a polarizing beam splitter (PBS). A subsequent half-wave plate (HWP) and PBS were used to direct a fraction of the light to the setup. Half of that light was split off by beam splitter BS1 and was brought to interference with light from frequency-stabilized reference laser M2 on photodetector PD2. A frequency counter (SR620 by Stanford Research Systems) and a computer were used to measure the difference frequency. The other half of the beam was used for frequency stabilization to an optical cavity. Here, the Pound-Drever-Hall scheme was used [8]. The light was transmitted through an electro-optical modulator that imposed $10 \mathrm{MHz}$ sidebands onto the beam. The light was directed through a PBS and a quarter-wave plate (QWP) and coupled into a stable reference cavity. The reference cavity consisted of a cylindrical spacer made of ultralow-expansion ceramic (ULE) by Corning with a diameter of $8 \mathrm{~cm}$, a length of $21 \mathrm{~cm}$ (freespectral range of $714 \mathrm{MHz}$ ), and a central bore of $1 \mathrm{~cm} \mathrm{di-}$ ameter. Two small bores were drilled perpendicular to the central bore for evacuation. A plane mirror and a concave mirror with $-1 \mathrm{~m}$ radius of curvature were optically contacted to the ends of the spacer. The finesse measured 9400. The cavity was manufactured by Research ElectroOptics (USA), and it was placed in a vacuum chamber to remove heat transfer by convection. Heat transfer by radiation and conduction were reduced by thermal shields consisting of four gold-coated steel cylinders separated by ceramic spacers. The light reflected from the cavity was detected by photodetector PD1. Its signal was mixed with the local oscillator (LO) and low-pass filtered by a fourpole Tschebyscheff with a corner frequency of $1 \mathrm{MHz}$ to remove the sum frequency at $20 \mathrm{MHz}$. The resulting error signal was sent to a servo and fed back to the PZT actuator of the fiber laser. With a servo consisting of a variable gain stage, a two-pole low-pass filter at $7.5 \mathrm{kHz}$, a

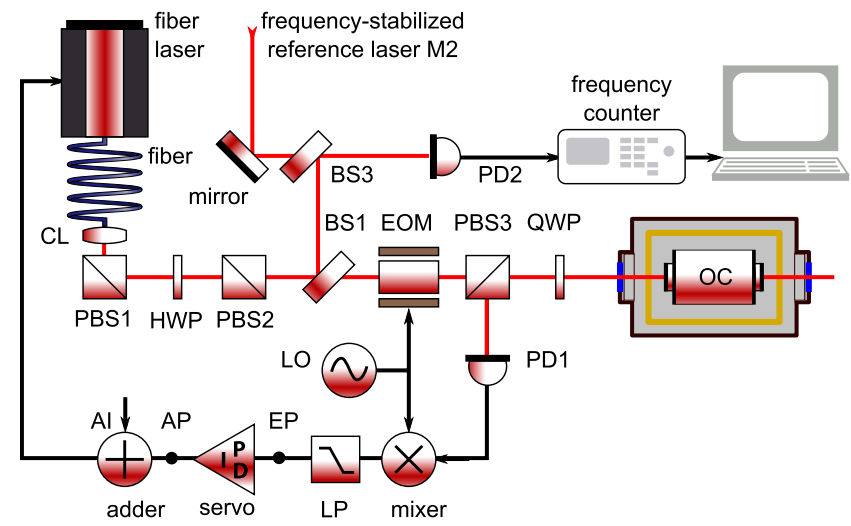

Fig. 1. (Color online) Frequency-stabilization setup for the fiber laser; beam splitter, BS; collimating lens, CL; electro-optical modulator, EOM; low-pass filter, LP; photodetector, PD; quarterwave plate, QWP; optical cavity, OC; adder input, AI; actuator point, AP; error point, EP; figure drawn using [17]. damped integrator to $1.7 \mathrm{kHz}$, and a switchable damped integrator up to $100 \mathrm{~Hz}$, a unity gain frequency up to $2.4 \mathrm{kHz}$ was achieved. Reference laser M2 was frequency stabilized using an identical but independent setup, which used $12 \mathrm{MHz}$ sideband frequency and a slightly different servo. Further details are given in [18].

Figure 2 shows measured frequency noise of the fiber laser and the reference NPRO M2 shown in Fig. 1 (Mephisto 500 by Innolight, operated at $300 \mathrm{~mW}$ output power). The blue trace labeled "FL Y10 beat" shows the free-running frequency noise of the fiber laser emitting $1 \mathrm{~W}$. It was obtained from a difference-frequency measurement between free-running fiber laser and frequencystabilized reference NPRO laser. The difference frequency was measured with a frequency counter (SR620 by Stanford Research Systems), and the program lpsd (logarithmic power spectral densities), which is optimized for spectrum and spectral density estimation from long time series by Fourier transforms [19,20], was used to calculate spectral densities.

The red trace labeled FL Y10 shows again the freerunning frequency noise of the fiber laser emitting $1 \mathrm{~W}$. It was recorded as a consistency check and for measuring frequency noise at higher Fourier frequencies. Here, the frequency noise of the fiber laser was measured by stabilizing its frequency to a stable optical resonator and reading out the actuator signal for Fourier frequencies below unity gain frequency and the error signal for higher Fourier frequencies. For a stable optical resonator and a stable frequency lock, the frequency actuator has to counteract the free-running frequency noise. Thus, for Fourier frequencies below the stabilization bandwidth, one can read the free-running frequency noise from the actuator signal. For Fourier frequencies above the stabilization bandwidth, the control loop cannot counteract disturbances anymore, and the error signal shows the remaining, uncorrected frequency noise of the laser.

Up to $2 \mathrm{kHz}$, the red trace labeled "FL Y10" was obtained from the calibrated actuator signal (for actuator signal calibration see Section 3). The unity gain frequency measured $2.4 \mathrm{kHz}$. For higher Fourier frequencies, the calibrated error signal was plotted when the fiber laser was frequency locked with a unity gain frequency of

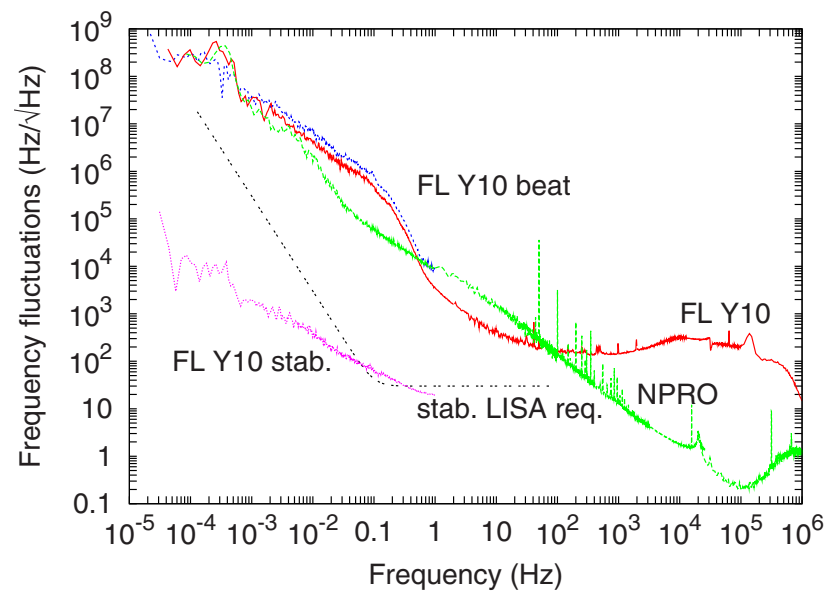

Fig. 2. (Color online) Measured free-running and stabilized frequency noise of fiber laser. 
$500 \mathrm{~Hz}$. Since the error signal is low-pass filtered by the high-finesse cavity, it was divided by an equivalent simple pole (the "cavity pole") at the pole frequency of $38 \mathrm{kHz}$ that was obtained from separate measurements [[18], p. 73]. For Fourier frequencies up to $2 \mathrm{kHz}$ an analog-to digital converter in a computer and lpsd were utilized; for higher frequencies up to $100 \mathrm{kHz}$, an FFT spectrum analyzer (SR 785 by Stanford Research Systems) was used, and for frequencies between $100 \mathrm{kHz}$ and $1 \mathrm{MHz}$, an $\mathrm{RF}$ spectrum analyzer (Agilent 4396B) was used.

For comparison, the free-running frequency noise of the reference NPRO (M2) has been measured as well and is shown as green trace labeled NPRO. Reference laser M2 was operated at $300 \mathrm{~mW}$ output power, and the green trace has been combined from three separate measurements: (1) Up to Fourier frequencies of $1 \mathrm{~Hz}$, the trace was obtained from a beat measurement between freerunning M2 and an identical NPRO called M1 replacing the fiber laser in Fig. 1. M1 used the same frequency stabilization setup as described above for the fiber laser. For higher Fourier frequencies, the frequency counter could not be read fast enough. Instead, M2 was frequencylocked to a stable cavity, and actuator and error signals were read out. (2) From $1 \mathrm{~Hz}$ to $25 \mathrm{kHz}$, the calibrated actuator signal was measured while M2 was frequencylocked with a unity gain frequency of $25 \mathrm{kHz}$. (3) For the remainder of the green trace, M2 was locked with $16.6 \mathrm{kHz}$ unity gain frequency, and the calibrated error signal was measured. The data plotted was divided by the equivalent cavity pole as it has been discussed above for the red trace labeled FL Y10. Since reference laser M2 was frequency-stabilized to a different reference cavity than the fiber laser, the corresponding pole frequency of $149 \mathrm{kHz}$ was used. The sharp peak at $316.5 \mathrm{kHz}$ in the green trace labeled NPRO is the relaxation oscillation of the NPRO; the peaks at $50 \mathrm{~Hz}$ and multiples stem from the electrical power grid.

The good overlap of the blue and the red trace ("FL Y10 beat" and "FL Y10") in Fig. 2 shows that the two different measurement methods for the free-running frequency noise of the fiber laser show consistent results. Compared to the free-running frequency noise of the NPRO, the noise of the fiber laser is higher up to a factor of 20 for frequencies below $0.5 \mathrm{~Hz}$. For Fourier frequencies above $100 \mathrm{~Hz}$ the free-running frequency noise of the fiber laser is up to three orders of magnitude higher than the NPRO frequency noise. For Fourier frequencies between $0.5 \mathrm{~Hz}$ and $100 \mathrm{~Hz}$, the fiber laser frequency noise is up to a factor of 5 below the NPRO frequency noise.

The pink trace labeled "FL Y10 stab." was obtained from a beat measurement between frequency-stabilized laser M2 and frequency-stabilized fiber laser, and it gives an upper limit to the frequency noise of both lasers. Due to the use of a frequency counter with $0.5 \mathrm{~s}$ gate time, measurements were limited to frequencies below $1 \mathrm{~Hz}$. The noise of the pink trace (FL Y10 stab.) is due to the uncorrelated noise contributions from the two independent frequency references and stabilizations (including temperature fluctuations of the cavities, noise of the stabilization systems, and residual amplitude modulations from the electro-optical moduators).

The pink trace (FL Y10 stab.) demonstrates that the stabilized-fiber laser setup complies with the LISA requirement for the stabilized laser frequency noise (black dotted trace labeled "stab. LISA req.," assuming armlocking [21]) within the LISA science bandwidth $(0.1 \mathrm{mHz}-1 \mathrm{~Hz})$. We calculated that the demonstrated frequency stabilization bandwidth allows sufficient noise suppression to meet the LISA frequency stability requirement up to $100 \mathrm{~Hz}$. At frequencies higher than $100 \mathrm{~Hz}$, the frequency noise of the stabilized fiber laser is up to three orders of magnitude higher than that of the NPRO. This out-of-band noise could potentially fold back into the measurement bandwidth of LISA due to aliasing in the LISA phasemeter. We have verified that the decimation filter of the current LISA phasemeter design [22] is compatible with the measured frequency noise, although this issue remains to be experimentally demonstrated in future work. In order to optimize the control loop, knowledge of the frequency actuator characteristics is required.

\section{PZT ACTUATOR EFFICIENCY AND TRANSFER FUNCTION}

In order to measure the PZT actuator efficiency, the laser frequency was brought close to a resonance frequency of the stable optical cavity shown in Fig. 1 by temperature adjustment of the fiber laser. A triangular voltage ramp was applied to the PZT so that the laser frequency was swept over a resonance frequency of the cavity. When either the carrier frequency or the sidebands passed through the cavity resonance frequency, error signals were produced. From the known frequency spacing of the two sidebands $(20 \mathrm{MHz})$ and the measured voltage that was required to shift the laser frequency, the PZT efficiency was determined. The measurement was performed seven times and the results were averaged. An average small-signal PZT tuning coefficient of 12.1 $\mathrm{MHz} / \mathrm{V} \pm 0.5 \mathrm{MHz} / \mathrm{V}$ was measured.

Due to the demanding requirements for laser frequency stability in the LISA mission, the frequencies of the LISA lasers will be actively stabilized. For an optimized control loop design, in addition to the "static" frequency tuning coefficient, the knowledge of the dynamic frequency response of the laser frequency actuator is required. It can be extracted from the transfer function from actuator point (AP) to error point (EP) shown in Fig. 1. In addition to the actuator characteristics, this transfer function also contains the pole caused by the reference cavity as described in the preceding section. This pole frequency has been measured as $38 \mathrm{kHz}$ in an independent measurement [[18], p. 73], corresponding to a reference cavity linewidth of $76 \mathrm{kHz}$ (FWHM).

An error signal for frequency stabilization can only be obtained when the laser frequency is within the cavity linewidth, which is not the case when the laser frequency is free running. Hence, in order to measure the frequency response of the frequency actuator, the laser frequency was locked to the cavity as shown in Fig. 1 using the Pound-Drever-Hall stabilization scheme. While the laser frequency was locked, a sinusoidal disturbance signal was inserted into the loop at the adder input (AI in Fig. 1, and its transfer from actuator point AP to error point EP was measured. The resulting transfer function is shown in 


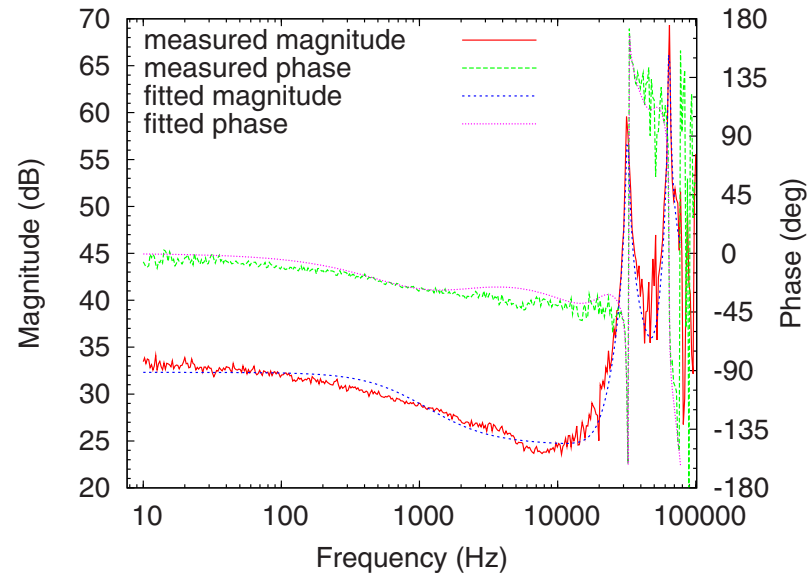

Fig. 3. (Color online) Transfer function of PZT frequency actuator; measurement and fitted function.

Fig. 3. In addition to the measured data, Fig. 3 shows a fit function. The most important parameters are the frequency and quality factor of the first PZT resonance, $32 \mathrm{kHz}$, and 26, respectively, and the delay of $20 \mu \mathrm{s}$.

With this information, we have designed, but not built, an optimized control loop. When accepting only $20^{\circ}$ phase margin, $4 \mathrm{kHz}$ unity gain frequency should be possible with a loop filter consisting of an integrator and a complex pole at $10 \mathrm{kHz}$ with a quality factor of 0.8 . We assume that different samples of the fiber laser will show some variability in frequency and quality factor of the PZT resonance due to fabrication details.

\section{CONCLUSION}

In conclusion we have investigated the suitability of an ytterbium-doped distributed-feedback fiber master oscillator power amplifier system emitting $1 \mathrm{~W}$ for the spaceborne interferometric gravitational-wave detector LISA. For Fourier frequencies up to $100 \mathrm{~Hz}$, the free-running frequency fluctuations were comparable to those of a nonplanar ring oscillator. For higher Fourier frequencies, the fiber laser system showed up to 3 orders of magnitude higher frequency fluctuations. The frequency actuator was characterized, and a stable frequency lock to an optical resonator meeting LISA's requirements was demonstrated. In the complete LISA measurement band, the frequency noise measurement of the fiber laser directly showed that the laser is compatible with the LISA phase measurement system. The measured out-of-band laser frequency noise should be compatible with the current design of the LISA phasemeter, which remains to be demonstrated experimentally in future work.

Any laser to be used for LISA also needs to be spacequalified, with the main issues being vacuum compatibility, radiation hardness, reliability, lifetime, and operating temperature range. These issues have not yet been addressed.

\section{ACKNOWLEDGMENTS}

We gratefully acknowledge support by Deutsches Zentrum für Luft- und Raumfahrt (DLR) (reference $50 \mathrm{OQ}$ 0601).

\section{REFERENCES}

1. K. Danzmann and A. Rüdiger, "LISA technology-concepts, status, prospects," Class. Quantum Grav. 20, S1-S9 (2003).

2. T. J. Kane and R. L. Byer. "Monolithic, unidirectional single-mode Nd:YAG ring laser," Opt. Lett. 10, 65-67 (1985).

3. I. Freitag, A. Tünnermann, and H. Welling, "Power scaling of diode-pumped monolithic Nd:YAG lasers to output powers of several watts," Opt. Commun. 115, 511-515 (1995).

4. LISA: System and Technology Study Report, ESA document ESA-SCI 11, 2000.

5. B. S. Sheard, M. B. Gray, D. E. McClelland, and D. A. Shaddock, "Laser frequency stabilization by locking to a LISA arm," Phys. Lett. A 320, 9-21 (2003).

6. S. L. Gilbert, "Frequency stabilization of a tunable erbiumdoped fiber laser," Opt. Lett. 16, 150-152 (1991).

7. N. Park, J. W. Dawson, and K. J. Vahala, "Frequency locking of an erbium-doped fiber ring laser to an external fiber Fabry-Perot resonator," Opt. Lett. 18, 879-881 (1993).

8. R. W. P. Drever, J. L. Hall, F. V. Kowalski, J. Hough, G. M. Ford, A. J. Munley, and H. Ward, "Laser phase and frequency stabilization using an optical resonator," Appl. Phys. B: Photophys. Laser Chem. 31, 97-105 (1983).

9. G. A. Cranch, "Frequency noise reduction in erbium-doped fiber distributed-feedback lasers by electronic feedback," Opt. Lett. 27, 1114-1116 (2002).

10. W. H. Chung, H. Y. Tam, M. S. Demokan, P. K. A. Wai, and C. Lu, "Frequency stabilization of DBR fiber grating laser using interferometric technique," IEEE Photon. Technol. Lett. 13, 951-953 (2001).

11. X. Zhang, N. H. Zhu, L. Xie, and B. X. Feng, "A stabilized and tunable single-frequency erbium-doped fiber ring laser employing external injection locking," J. Lightwave Technol. 25, 1027-1033 (2007).

12. G. A. Ball, G. Hull-Allen, and J. Livas, "Frequency noise of a Bragg grating fibre laser," Electron. Lett. 30, 1229-1230 (1994).

13. C. Spiegelberg, J. Geng, Y. Hu, Y. Kaneda, S. Jiang, and N. Peyghambarian, "Low-noise narrow-linewidth fiber laser at 1550 nm," J. Lightwave Technol. 22, 57-62 (2004).

14. J. Geng, J. Wu, S. Jiang, and J. Yu, "Efficient operation of diode-pumped single-frequency thulium-doped fiber lasers near $2 \mu \mathrm{m}$," Opt. Lett. 32, 355-357 (2004).

15. T. Rosenband, D. B. Hume, P. O. Schmidt, C. W. Chou, A. Brusch, L. Lorini, W. H. Oskay, R. E. Drullinger, T. M. Fortier, J. E. Stalnaker, S. A. Diddams, W. C. Swann, N. R. Newbury, W. M. Itano, D. J. Wineland, and J. C. Bergquist, "Frequency ratio of $\mathrm{Al}+$ and $\mathrm{Hg}+$ single-ion optical clocks; metrology at the 17th decimal place," Science 319, 1808-1812 (2008).

16. N. R. Newbury, P. A. Williams, and W. C. Swann, "Coherent transfer of an optical carrier over $251 \mathrm{~km}$," Opt. Lett. 32, 3056-3059 (2007).

17. Alexander Franzen, Component library Version 3, 2006.

18. Michael Tröbs, "Laser development and stabilization for the spaceborne interferometric gravitational wave detector LISA," PhD dissertation (University of Hannover, 2005).

19. M. Tröbs, and G. Heinzel, "Improved spectrum estimation from digitized time series on a logarithmic frequency axis," Measurement 39, 120-129 (2006).

20. M. Tröbs and G. Heinzel, "Corrigendum to 'Improved spectrum estimation from digitized time series on a logarithmic frequency axis'," Measurement 39, 120-129 (2006); 142, 170 (2009).

21. H. R. Schulte, P. F. Gath, and M. Herz, "Laser frequency stabilization by using arm-locking," in Proceedings of 6 th International LISA Sympostion (AIP, 2006), pp. 379-383.

22. D. Shaddock, D. Ware, P. G. Halverson, R. E. Spero, and B. Klipstein, "Overview of the LISA phasemeter," in Proceedings of 6th International LISA Sympostion (AIP, 2006), pp. 654-660. 